\title{
Depressão na gravidez e prematuridade. Aspectos epistemológicos da investigação
}

\author{
Depression during pregnancy and preterm delivery. \\ Epistemological aspects in research
}

Marcia Zucchi 1

1 Rua Barata Ribeiro 250, sala 3, Rio de Janeiro, RJ 22040-000, Brasil. marciazucchi@hotmail.com
Abstract This paper examines recent research on the association between depression during pregnancy and preterm delivery. Dissemination of scientific information on this disease entity (depression) means that it is often present in common-sense discourse, al though frequently used rather imprecisely. Whi le various areas from the mental health field approach depression in very different ways, they suggest the influence of emoti onal states on organic outcomes. Still, this relationship remains difficult to prove by conventional scientific research methods. One problem in establishing associations between depression and preterm delivery appears to be due to epistemological aspects related to the theoretical demarcation of depression.

Key words Depression; Pregnancy; Prematurity; Premature Infant; Epistemology

Resumo Este trabalho analisa pesqui sas recentes que estudam a associação entre depressão na gravidez e prematuridade. A difusão de informações científicas faz dessa categoria nosográfica (depressão) uma presença constantee, muito freqüentemente, imprecisa, nos di scursos do senso comum. No campo da saúde mental, são várias as áreas que a abordam e de modos bastante diversos. Por outro lado, apesar de haver indi cações sugerindo a influência de estados emocionais em desfechos orgâni cos, permanecem dificuldades em demonstrá-la através dos métodos convencionais de pesquisa ci entífica. Parece que um dos problemas em associar depressão à prematuridade deve-se a aspectos epistemológicos rel acionados à demarcação teórica da depressão. Palavras-chave Depressão; Gravidez; Prematuridade; Prematuro; Epistemologia 


\section{Introdução}

O foco central deste trabalho é a depressão na gravidez e sua possível implicação na ocorrência de partos prematuros. A própria circunscrição do quadro clínico de depressão na gravidez, no entanto, sofrerá alterações profundas conforme a perspectiva teórica do pesquisador. O intuito deste estudo é destacar al gumas dificuldades de delimitação desse quadro nosográfico, bem como apresentar e discutir as conclusões de algumas pesquisas que buscam associar depressão na gravidez e parto prematuro, considerando-se que este último permanece sendo um dos principais problemas de saúde pública perinatal.

Seja como entidade nosográfica, seja como experiência fenomenológica, a depressão adquire interesse aqui, tanto por seu 'poder definidor' ou 'poder explicativo' no que diz respeito ao senso comum (o termo depressão é utilizado para representar quase toda sorte de estados de 'dor psíquica'), como pela freqüência com que tem sido associada aos mais diversos quadros clínicos, tornando complexas suas explicações etiológicas, bem como sua terapêutica. A definição de depressão em termos científicos é, no entanto, bastante problemáti$\mathrm{ca}$, conforme discutiremos ao longo do texto.

Sabe-se que os fenômenos depressivos são objeto de diferentes áreas do saber - as psicologias, as psiquiatrias, as psicanálises, as ciências sociais, além da genética, da neurologia e outras -, cujos contornos são, por vezes, muito pouco definidos. Nenhuma delas é hegemônica em si, nem tampouco no domínio do saber sobre o mental ou psíquico - este objeto pouco preciso, complexo ou 'pluriobjeto'. Nesse sentido, são inevitáveis as interseções e interfaces (psiquiatria psicanalítica, psiconeuroimunologia, psicobiologia, neuropsiquiatria etc.), nas quais os conceitos se organizam em sintaxes que se diferenciam tanto das fontes originais (uma psicologia ou uma psiquiatria específicas), quanto das novas combinações de saberes.

Além da abundância de literatura científica, nas mais diversas tendências, acerca do tema depressão, há, também, uma profusa literatura não científica sobre o tema. Este é presença freqüente na imprensa escrita, falada ou televisiva; comparecendo nos discursos dos representantes de qualquer classe social (pelo menos nos países cuja cultura é ocidentalizada); atualmente, acompanha-se, inclusive, sua apresentação em home pages de redes computacionais. Na última década, a circulação das informações científicas tem se dado de forma ampla, independente da qualidade destas ou do quanto de incerteza escamoteiam. O senso comum vem se construindo sob esta forte influência da divulgação dos saberes científicos (Granger, 1993:16-19).

\section{Alguns aspectos epistemológicos da conceitualização da depressão}

Tratar de aspectos epistemológicos é, antes de mais nada, tratar da lógica sob a qual um conhecimento se processa. Se o conhecimento só se faz por mediação, a razão é o mediador que caracteriza o conhecimento científico. Morin descreve a razão como: “um método de conhecimento baseado no cálculo ena lógica (na origem, ratio quer dizer cálculo), empregado para resolver problemas postos ao espírito, em função dos dados que caracterizam uma si tuação ou um fenômeno. A racionalidade éo estabelecimento de uma adequação entre uma coerência lógica (descrita, explicativa) e uma realidade empírica" (Morin, 1990:121). Compreender os périplos da noção de depressão implica reconhecer a racionalidade do uso deste conceito em diferentes contextos teóricos.

O estudo da depressão coloca o pesquisador - clínico ou teórico - em confronto com problemas epistemológicos como o da multiplicidade de definições de depressão conforme os referenciais teóricos utilizados. A escolha de tais referenciais tem implicações no campo tanto teórico, quanto prático.

Do ponto de vista epistemológico, é importante se ressaltar a diferença de recorte do objeto mente ou psiquismo, sede da depressão. Conceber a depressão como ocorrência de um aparelho psíquico forjado no embate entre moções pulsionais e pressões culturais é completamente diferente, por exemplo, de compreendê-la como efeito de processos bioquímicos num aparato neuronal. Para as neurociências, o mental se circunscreve nos processos de cognição e nas estruturas cerebrais (circuitos neuronais, bioquímica cerebral) (Andreasen, 1997:1.586), enquanto, nos saberes psicodinâmicos, a ênfase do mental está na subjetividade; portanto, na organização particular, simbólica, do que quer que seja o mental (neurônios, genes, relações sociais ou traços de linguagem). Nesse sentido, a categoria ou conceito depressão sofre os efeitos lógi cos de estar vinculada a uma ou outra concepção do mental. Logo, não seria necessário demonstrar as enormes diferenças na clínica da depressão conforme o modelo teórico que se utilize.

Se é possível relacionar o mental orgânico com o mental simbólico, como pretendem, es- 
pecialmente, os pesquisadores das neurociências (Andreasen, 1997:1.586), é necessário que se considerem, antes, os ganhos e as perdas que tal junção pode implicar. Essas relações só se fazem através de reduções, que, por vezes, custam a perda do objeto em si (Samaja, 1992: 15). Por outro lado, a finalidade pode justificar esta tentativa. A clínica do mental (e suas dificuldades) é, sem dúvida, um forte estimulante para a criação destas interfaces, porém aqui também (clínica do mental) há diferenças marcantes: umas caminhando no sentido da eliminação ou controle dos sintomas e transtornos; outras, considerando os sintomas como discursos subjetivos, cuja decisão sobre seu destino (eliminação ou não) não se deve ao profissional que conduz a clínica, nem é possível para ele.

Não se pretende, aqui, priorizar qualquer dos enfoques acima descritos. Deseja-se, em especial, ressaltar a mudança de perspectiva de um fenômeno (a depressão, no caso) quando vinculado a um ou outro desses objetivos. E estes objetivos, ou sentidos da intervenção clínica, têm sua determinação (ao menos em parte) no privilégio à vertente subjetivante ou objetivante da produção de conhecimento. O objetivismo poderia ser sintetizado a partir das proposições de Lakoff \& Johnson (1980), como uma visão do mundo constituído de objetos com características e propriedades independentes dos seres que com eles se relacionam, possibilitando, assim, seu conhecimento verdadeiro, por meio de métodos e linguagem claros e objetivos, como pretendem ser o método e a linguagem científicos. Já o subjetivismo compreende o conhecimento dos objetos do mundo mediante as relações entre os seres e os objetos, enfatizando todas as formas de manifestação - arte, poesia etc. - como formas de conhecimento.

Outra questão epistemológica relevante na análise de um conceito é a dificuldade em se rastrearem as semelhanças ou linhas de continuidade internas a um saber, ou entre saberes diversos, no que tange ao uso desse conceito, em razão das mudanças de estatuto que este sofre no seu contexto de uso. A depressão, por exemplo, tem sido tratada, ora de modo substantivo ora adjetivo. Conforme descreve Palmeira, em sua tese de mestrado acerca das relações entre psique e câncer: “(...) em alguns casos a 'depressão' é entendida como al go que o sujeito sofre, em outros é interpretada como algo inerente à própria natureza do sujeito" (Palmeira, 1994:47).

Uma tentativa de neutralização deste problema tem sido feita pela epidemiologia psi- quiátrica com seus sistemas de classificação, nos quais estados como os de depressão seriam sempre adjetivos, o resultado de um somatório de sinais. (Discutir-se-ão os sistemas classificatórios adiante.)

Na perspectiva mais estritamente neurobiológica, a depressão é associada a fatores tais como: alterações de processos cerebrais adaptativos (Andreasen, 1997:1.588); a intercorrências nos sistemas de transmissão noradrenérgicos do sistema nervoso central (s.n.c.); ao processo de recaptação da serotonina ao nível sináptico do referido sistema (s.n.c.); ou ainda à deficiência de dopamina no sistema nigroestriatal (Simões et al., 1996:4-5). Outra vertente da pesquisa biomédica associa a depressão a fatores hormonais, como o estrogênio, por sua "ação direta e indireta sobre os neurônios do s.n.c." (Simões et al., 1996:5), condição considerada como uma das prováveis responsáveis pela freqüência de depressão, duas vezes maior, nas mulheres do que nos homens (Paykel, 1991). Fica evidenciado que a depressão, independente da hipótese que a explique, é compreendida como um fenômeno adjetivo, resultante de processos neurobiológicos ou genéticos.

Os modelos de produção de conhecimento acerca da depressão, nestas áreas, são objetivos. Seja o que for, supõe-se que a depressão seja exterior àquele que a pesquisa.

A depressão como experiência vivida - subjetiva - é tratada pelos saberes psi codinâmicos (incluindo aqui os culturais). Seria temerário, entretanto, conceber qualquer universalidade nas formas de pensar a depressão pelas diversas correntes de saber que têm as experiências psíquicas como seus objetos.

Para os saberes teóricos que descrevem a subjetividade e suas manifestações (as psicanálises, as antropologias etc.), a depressão também tende a ser vista de modo adjetivo, como resultante de processos agora não mais biológicos, mas psíquicos ou sociais. A pretensão do conhecimento objetivo referente a essa experiência é abandonada e substituída por uma racionalidade que não supõe encontrar a verdade do fenômeno depressivo, e sim defrontarse com sua(s) manifestação(ões) e seus limites.

Referindo-se às diferenças de racionalidade do saber científico e do saber filosófico (não positivista), Atlan faz afirmações que podem ser úteis para esclarecer a racionalidade dos saberes psicodinâmicos: “Assim, contrariamente ao ideal das filosofias neopositivistas, que procuravam imitar a física e a sua forma lógico-matemática, o papel da filosofia seria falar daquilo que não pode ser formalizado, utilizar uma linguagem natural, com assuas metáforas, 
as suas anal ogias ea indefini ção que as acompanha, sem, por isso, renunciar a continuar racional; e para tal, distinguir as boas das más anal ogias, as metáforas enriquecedoras das metáforas enganadoras, o pouco vago, que oculta o que deveria ser dito, do demasiado vago, potencial de criação" (Atlan, 1991:101).

A organização dos saberes sobre o mental em sistemas classificatórios: algumas implicações clínicas

Conforme apresentado no prefácio à edição brasileira da Classificação Internacional de Doenças. Descrições Clíni cas e Diretrizes Diagnósticas - CID 10 - da Organização Mundial de Saúde (OMS) (OMS, 1993:XI), o esforço sistemático de classificação dos transtornos mentais, orientado por esta entidade, data da década de 60 e vem se ampliando e se especificando desde então.

Esta última versão de 1992, junto com o Diagnostic and Statistical Manual of Mental Disorders (DSM IV) (American Psychiatric Association, 1994), representa os resultados mais recentes desta tendência taxonômica, a qual vem buscando instrumentos que permitam a comunicação entre os diferentes profissionais envolvidos na clínica, na pesquisa e na educação em saúde mental.

Tais classificações se caracterizam pela descrição de sinais e sintomas, com um declarado abandono da noção de doença mental e a opção pelos conceitos de episódios e transtornos. Este último é definido como "um conjunto de sintomas ou comportamentos, clinicamentere conhecível, associado, na maioria dos casos, a sofrimento e interferência com funções pessoais" (OMS, 1993:V). Outros dois eixos utilizados nas classificações são: a distribuição dos sinais e sintomas no tempo (episódica ou recorrente) e sua intensidade (grave, moderada ou leve).

Esta opção pelo modelo sindrômico em detrimento do modelo nosológico se dá, especialmente, por causa da ausência de certezas quanto à etiopatogenia dos transtornos mentais e/ ou da complexidade que envolve a causalidade do psíquico. O objetivo apresentado para estas categorizações é: “mel horar o diagnósti co e a classificação dos transtornos mentais", facilitando a clínica, a pesquisa e a comunicação entre profissionais da área de saúde mental (OMS, 1993:XI). Por outro lado, dependendo da finalidade que se atribua a um diagnóstico, este esforço classificatório será de maior ou menor valor.
Zarifian (1989) define um diagnóstico como um instrumento que "permite comunicar acerca de um doente, (...) permite comparar grupos de pacientes entre si , (...), [não sendo necessário na clínica, entretanto], pois a abordagem é essencialmente intuitiva" (Zarifian, 1989:4547). Nesta mesma linha, Lajeunesse (1989) afirma que: “o interesse de um diagnóstico fiel eváli do é condensar uma informação com virtude prognóstica e, por conseguinte, condicionar a orientação terapêuti ca" (Lajeunesse, 1989:72). Segundo este autor, tal fidelidade se obtém às custas da retirada das sintomatologias puramente subjetivas, quando da descrição das categorias, procedimento que, para ele, não garante a objetividade e, ainda, acirra a dicotomia entre os praticantes da clínica do mental (subjetivistas e objetivistas).

O abandono da posição nosográfica, nos dois sistemas classificatórios supracitados, deve-se, também, a uma opção pela não-utilização de qualquer sistema teórico específico, no bojo, ainda, de um projeto de objetividade de tais classificações. Se, por um lado, esta postura 'suprateórica' gera a clareza dos sintomas descritos, por outro, multiplica-os de forma progressiva, dificultando sua utilização clínica como instrumento de projeto terapêutico (o capítulo dos transtornos mentais do CID 9 tinha trinta categorias, a atual versão - CID 10 tem cem).

Quanto à categoria depressão, os organizadores da CID 10 advertem que esta versão ainda é fonte de muita discordância entre psiquiatras. Supõem, entretanto, que tais discordâncias serão dirimidas com “medidas fisiológicas e bioquímicas, ao invés (...) descrições clínicas de emoções e comportamentos" (OMS, 1993:XIII).

Uma perspectiva nosológica implica modelos teóricos de interpretação dos eventos. A 'costura' dos dados parece fundamental na própria caracterização dos fenômenos. Esta ausência de organização teórica dos sintomas leva, por vezes, a situações bizarras, na quais qualquer sujeito pode ser incluído sob certos diagnósticos. O transtorno depressivo é um caso exemplar deste tipo. Na classificação do DSM IV, a depressão é classificada como um transtorno afetivo do humor, que ocorre em um período mínimo de duas semanas, envolvendo episódios depressivos e mais, pelo menos, quatro dos seguintes sintomas: queixas de tristeza, desesperança, perda de prazer generalizada, perda de apetite, perturbações do sono, alterações psicomotoras, diminuição de energia, sentimentos de desvalia ou culpa e pensamentos suicidas (American Psychiatric Association, 1994:323-329). Seria quase impossível 
encontrar um adulto ocidental que não se enquadra nesses critérios, especialmente quando se trata daqueles que vivem nos grandes centros urbanos.

Em relação aos critérios fisiopatológicos, bioquímicos ou genéticos como fontes de especificação diagnóstica, é preciso que se destaque a subversão que tal caminho pode criar na compreensão (e conseqüente abordagem) dos fenômenos mentais. Sobre isso, Zarifian (1989) afirma: “Os psicotrópicos tiveram papel importante, enão inocente, na evolução dos conceitos diagnósticos. Responsável por isso é sobretudo o marketing farmacêutico. (...) Se bem que os psicotrópicos não sejam senão tratamentos si ntomáti cos enão específicos de uma afecção mental, o cenári o está armado. As cl assi fi cações de psicotrópicos reforçam a si tuação. Há antipsicóticos, anti depressivos e ansiolíticos: então há psicoses, depressões e ansiedade" (Zarifian, 1989:49-50).

Poder-se-ia objetar que as versões atuais dos sistemas classificadores minimizam este problema abrindo mão das grandes categorias nosográficas. Todavia, a intenção de objetivação através de critérios estritamente biológicos permanece, como foi mostrado anteriormente. Conta-se, atualmente, com instrumentos poderosos para esses fins: além da fidedignidade da bioquímica, desfruta-se agora da precisão e do rigor das imagens (tomografia de emissão de pósitrons, por exemplo). Mas ainda corre-se o risco de identificar fenômenos de uma esfera (os afetos deprimidos, por exemplo) com achados de outra esfera (as possíveis al terações de imagens tomográficas quando de estados afetivos deprimidos), incorrendo em inevitável engano e confusão, com evidentes conseqüências clínicas.

Outra fonte de dificuldades no estabelecimento e uso clínico de um sistema classificador são as diferenças culturais. Estas são reconhecidas nos dois sistemas a que estamos nos referindo (DSM IV e CID 10), porém, em virtude da dificuldade para transformá-las em variáveis indicáveis objetivamente, elas não são consideradas. Mais uma vez, isso não ocorre sem prejuízo clínico. A esse respeito, Lutz (1985) afirma: "Argumentarei, entretanto, que a distinção entre o o quê e o como da experiência depressiva (...) somente faz sentido no contexto cultural Euro-Americano dentro do qual foi desenvolvido(...) O que é mais notável na visão ocidental da depressão éa afirmação implícita do caráter de oposi ção à al egria, ou pel o menos aos afetos positivos, em relação a um estado normal. (...) O queé particularmente desviante nos deprimidos ésua desistência em buscar a fe- licidade ou o amor desi, consi derados objetivos bási cos e normais das pessoas. Estas metas aparentemente naturais são, de fato, moldadas cultural mente, em contraste com outras possí veis definições de normalidade nas quais, por exemplo, a ênfase pode ser posta no cuidado de crianças ou parentes, ou em vivenciar emoções de caráter moral, corretas porém não prazerosas..." (Lutz, 1985:63-70).

Parece, então, que, se essa objetivação das classificações diagnósticas traz vantagens, tais como, a abertura de espaço para a consideração de configurações complexas, no interior de sistemas dinâmicos (Bastos \& Castiel, 1994:108), traz também alguns riscos clínicos, como o de se confundirem "os si gnos recol hi dos com a realidade da doença" (Clavreul, 1983:202). O fato de os sistemas classificadores terem abandonado a concepção de doença não significa que as pessoas (o senso comum) a tenham abandonado também, muito pelo contrário. Prova disso são os serviços clínicos cada dia mais lotados de autodenominados doentes do pânico ou deprimidos.

\section{Depressão na gravidez e prematuridade}

A prematuridade ou nascimento pré-termo, definido como o que ocorre com 36 semanas ou menos de gestação, é um dos principais problemas da perinatologia, respondendo por $70 \%$ das mortes neonatais (J. M. A. Lopes, comunicação pessoal). Em termos de saúde coletiva, a prematuridade associada ao baixo peso ao nascer tende a ser considerada uma questão de muita importância em razão de sua significativa prevalência - em torno de $10 \%$ - em quase todo o mundo, a despeito das diferenças de níveis de desenvolvimento entre os países (L. G. P. Silva, comunicação pessoal). Segundo Orr \& Miller (1995:165), o nascimento pré-termo e o baixo peso ao nascer são as principais causas de morbi-mortalidade infantil nos Estados Unidos, por exemplo.

A questão da prematuridade é relevante não só pelos índices de mortalidade a ela associados, mas também pela qualidade de vida restrita de muitos dos que a ela sobrevivem. Embora os avanços tecnológicos permitam a sobrevivência de crianças cada vez menores (em termos tanto de idade gestacional, como de peso ao nascer), as seqüelas dessa prematuridade podem ser extremamente graves e penosas, seja para criança, seja para sua família. Os dilemas éticos com os quais a clínica da prematuridade se vê envolvida, por vezes, deve impulsionar as pesquisas no sentido de sua redução. 
Com o intuito de diminuir estes índices, vários estudos epidemiológi cos têm sido desenvolvidos, pesquisando fatores de risco para prematuridade, tais como: fatores sócio-demográficos, idade materna (abaixo de vinte anos), grau de pobreza, condições de saúde da gestante (hipertensão, problemas de placenta, infecções), comportamentos da gestante (especialmente o uso de cigarro, drogas e álcool), falta ou inadequação do acompanhamento pré-natal (Orr \& Miller, 1995:165; L. G. P. Silva, comunicação pessoal). De acordo estes autores, no entanto, tais fatores respondem apenas por uma parte (em torno de $30 \%$ ) dos nascimentos prematuros, o que leva a pesquisa dos fatores de risco para a prematuridade em direção aos aspectos psicossociais (dentre outros).

Estudos prospectivos, como os de Victora et al. (1996), realizados no sul do Brasil, demonstram que a melhora nas condições sócio-econômicas, educacionais e nutricionais das gestantes não são suficientes para redução do baixo peso ao nascer (o qual, por sua vez, apresenta forte relação com a prematuridade e o crescimento intra-uterino retardado).

Um dos fatores psicológicos que tem sido pesquisado em sua possível associação à prematuridade, especialmente na última década, é a depressão na gravidez. Numa revisão da literatura nesse período, realizada por intermédio do sistema de pesquisa eletrônica Medline, encontramos estudos que poderiam ser subdivididos em dois grandes grupos:

1) Aqueles que pesquisam os fatores de risco para depressão na gravidez.

2) Os que buscam associar a depressão como fator de risco para certos desfechos obstétricos, tais como a prematuridade, o baixo peso ao nascer, a irritabilidade do bebê (Zuckerman et al., 1990), ou mesmo a mortalidade neonatal (Bustan \& Coker, 1994).

Em relação aos fatores de risco mais freqüentemente associados à depressão na gravidez, encontram-se as dificuldades econômicas e a falta de parceiro ou de suporte familiar e social (Millán et al., 1990; Jadresic et al., 1993; Hobfoll et al., 1995; Séguin et al., 1995). Nesse sentido, a prevalência da depressão na gravidez é maior em grupos de mulheres de baixa renda, negras, com baixos níveis de escolaridade (Zuckerman et al., 1989; Orr \& Miller, 1995; Séguin et al., 1995; Copper et al., 1996).

Para os fins deste trabalho, é necessário, porém, maior atenção aos estudos que pesquisaram a associação entre a depressão e a prematuridade. Nestes estudos, o fator de exposição (depressão) é abordado com algumas variações: ora é visto como um quadro psicológi- co em si, ora como um dos elementos de um quadro mais amplo - o estresse. Encontram-se, ainda, estudos associando tensão psicológica (distress) materna e prematuridade. Tais diferenças são importantes, pois indicam recortes diversos de certas realidades psicológicas, o que fica também evidenciado pelo uso de instrumentos de coleta de dados (questionários), diferentes em cada estudo. A comparação entre estes trabal hos fica limitada por estas diferenças. Tentar-se-á ressaltar aqui como cada estudo tratou a variável psicológica em questão, de modo a que algumas comparações possam ser feitas.

Um estudo de Hedegaard et al. (1993), realizado no Departamento de Obstetrícia do Arthus University Hospital (Dinamarca), pesquisou a relação entre a tensão psicológica (distress) na 16ạ e na 30ạ semanas de gestação e a ocorrência de parto prematuro, numa coorte de 5.872 mulheres. A porcentagem de partos pré-termo foi de 3,6\% (197 casos). Só foi encontrada significância estatística entre distress na trigésima semana e parto prematuro.

Em outro estudo prospectivo realizado por Orr \& Miller (1995), foi pesquisada a associação potencial entre sintomas depressivos maternos e parto prematuro, numa população de 1.861 gestantes assistidas em quatro hospitais de Baltimore (Maryland, USA). A porcentagem de partos prematuros foi de $9,5 \%$. Os autores só encontraram associação, estatisticamente significativa, entre sintomas depressivos e prematuridade na população negra (afro-americana) e de baixa renda.

Num terceiro estudo prospectivo (Copper et al., 1996), foi pesquisada a relação entre estresse materno e prematuridade, numa população de 2.593 mulheres grávidas. Nesta pesquisa desenvolvida pelo National Institute of Child Health and Human Devel opment, há uma concordância com o estudo anterior no que se refere à associação entre estresse e parto prétermo especialmente na população negra e de baixa renda. A escala utilizada neste estudo é uma escala adaptada de cinco outros questionários que mediam ansiedade, auto-estima, controle (mastery), depressão e estresse. A variável estresse é aqui considerada uma entidade distinta da depressão. Analisando exclusivamente o item depressão em sua relação com prematuridade, não foi encontrada associação estatística significativa.

Noutra pesquisa desenvolvida por Peacock et al. (1995), através do Departamento de Ciências da Saúde Pública do St. George's Hospital Medical School (Londres), com 1.513 mulheres grávidas, os achados indicam significante rela- 
ção entre estresse materno e parto pré-termo. Neste estudo, contudo, a depressão está incluída no conjunto dos fatores psicológicos considerados estressores, ao contrário do apresentado no estudo anterior. Aqui, a depressão, o baixo nível sócio-econômico, a baixa escolaridade, a ausência de companheiro e a procura de serviços de apoio anterior à gravidez foram os itens avaliados e considerados associados ao parto prematuro. Numa publicação anterior (1993) acerca desta mesma pesquisa, enfocando, porém, especificamente a depressão e a ansiedade como fatores ligados à prematuridade, os autores afirmam que, embora indesejáveis, tais ocorrências (depressão e ansiedade) são pouco importantes na determinação de desfechos obstétricos como o parto pré-termo.

Algumas observações sobre tais estudos merecem ser sublinhadas:

- Todos os estudos citados apresentam um tratamento estatístico cuidadoso com análises de confounding, regressão logística e análise multivariada para fatores associados ao desfecho prematuridade. No entanto, a variável pesquisada (depressão) não é de simples definição ou facilmente redutível a elementos mensuráveis quantitativamente (Palmeira, 1994).

- Em nenhum dos estudos a associação entre depressão e prematuridade é direta e amplamente evidente. A depressão não é, em si, fator preditivo para parto pré-termo. Já o distress no final da gravidez e o estresse parecem ser fatores com algum valor preditivo para prematuridade. Utilizou-se o termo 'algum', uma vez que o distress e o estresse não estão associados à prematuridade em toda população de gestantes.

- Sejam os sintomas depressivos, seja o distress, ou o estresse, a associação significativa deste à prematuridade se apresenta para muIheres negras, de baixa renda, baixo nível de escolaridade, e estão, ainda, relacionados a comportamentos aditivos, como uso de álcool, fumo ou drogas (Zuckerman et al., 1989). Tais características agrupam um contingente social que responde pela maior prevalência tanto de depressão, quanto de prematuridade, isoladamente. Fica, portanto, difícil não só a avaliação dos efeitos complexos da pobreza e marginalização social na geração de experiências depressivas, como também a compreensão exata de como estes sentimentos poderiam contribuir para ocorrência de um parto prematuro.

\section{As dificuldades para se concluir...}

As principais críticas aos estudos de variáveis psicossociais têm sido em torno de questões como tamanho da amostra, modelo de estudo, validade do fator de exposição e falta de controle das variáveis de confusão (Hedegaard et al., 1993:237). Acredita-se, entretanto, que haja dificuldades anteriores relativas à construção e à operacionalização de conceitos teóricos.

A abordagem empírica de um conceito (a depressão, por exemplo) implica reduções como as descritas por Almeida Filho (1989): transformação do conceito em variável; desta, em indicador; e, finalmente, em medida. Nem sempre, no entanto, tais reduções são possíveis, sem que se cometa certa "infidelidade metodológi ca ao marco teórico do conceito" (Almeida Filho, 1989:81), ou, conforme indicado em item anterior, sem que se corra o risco da "perda do objeto em si" (Samaja, 1992). A depressão, assim como a maior parte dos conceitos relativos a fenômenos psíquicos, sofre tais alterações quando se tenta abordá-la numa relação estritamente objetiva.

As pesquisas envolvendo a categoria depressão demonstram, todavia, a dificuldade em isolar um quadro, como o da depressão, de outros, como o estresse ou o distress. Conforme demonstrado por Palmeira (Palmeira, 1994:4445), a depressão é tratada por certos autores como uma das muitas formas de tensão psíquica (distress); por outros, como uma dificuldade em lidar com o estresse emocional; para um terceiro grupo, a depressão e a ansiedade seriam, indistintamente, os sintomas do distress; outros, ainda, consideram esta junção inadequada, descrevendo depressão e ansiedade como quadros absolutamente distintos, e assim se sucedem as dificuldades de objetivação do psíquico. Referindo-se às restrições da utilização dos procedimentos epidemiológicos para verificação da relação entre depressão e câncer, o autor afirma: "A dificuldade para falar sobre 'depressão', por exemplo, é contornada pela introdu ção de especificidades que permitem definir a 'depressão endógena', a 'depressão ansi osa', a 'depressão psi cológi ca', a 'personalidade depressi va', o 'estado depressivo' ou, simplesmente, os 'si ntomas depressivos'.

Tal procedimento não resolve o problema (da apreensão do conceito), pois, como vimos, o que distingue um subtipo éaquil o que se agrega (a intensi dade, a duração, os el ementos associados) a um elemento central comum, que permanece inacessível na medida em que não é possível identificar um indicador (ou conjunto definido deindicadores) capaz de ocupar essa posi- 
ção (de el emento necessário). Trata-se de um exercício de adjetivação que não acrescenta nada de substantivo acerca do concei to original" (Palmeira, 1994:46).

No caso específico das pesquisas sobre a prematuridade, parece importante que se ressalte que a finalidade última destes trabalhos é clínica. Deste ponto de vista, tais pesquisas são úteis na organização do campo sintomático a ser abordado junto às pacientes obstétricas. $\mathrm{O}$ campo da clínica, no entanto, é o campo do singular, onde as manifestações, mesmo biológicas, ocorrem em conformidade a uma lógica particular à subjetividade de cada paciente.

A psicanálise tem contribuído para o campo da clínica médica por demonstrar o caráter erógeno (simbólico/subjetivo) do corpo e, portanto, de seu adoecimento. Desde a postulação freudiana em 1905 acerca da complacência somática - condição de alterabilidade das propriedades funcionais dos sistemas somáticos em conformidade com intenções psíquicas inconscientes -, os psicanalistas vêm buscando demonstrar, empírica e teoricamente, as relações entre processos psíquicos e manifestações somáticas. Sobre este assunto, ver Castiel (1994:59-88), onde o autor discute as diversas correntes da pesquisa psicossomática e suas correspondentes concepções dos modos de relação entre o somático e o psíquico.

Paralelo ao corpo biológico, o corpo vivido pelo sujeito humano é um corpo simbolizado e, por isso, prenhe de significações e sentidos. Tais significações 'jogam' tanto com a etiologia, quanto com o tratamento das doenças, participando destes.

Por outro lado, não é fácil (talvez nem possível) demonstrar tais relações por meio dos procedimentos preconizados pela ciência em seu modelo positivista. Podemos conhecer as significações específicas que um processo biológico assume para um indivíduo em particular; observamos a alteração de certas condições somáticas em seqüência a intervenções interpretativas num processo psicanalítico; não temos, porém, o domínio sobre cada passo desse processo.

Se a prematuridade for compreendida como uma ocorrência biológica, cuja si gnificação está associada a um conjunto de representações psíquicas de cada gestante em particular (suas representações sobre maternidade, fiIhos, família, feminilidade etc.), poder-se-ão depreender papéis muito específicos para a depressão em tal desfecho.

A passagem dos achados obtidos por instrumentos epidemiológicos (ciência do coletivo) para a clínica (ciência do particular) requer cuidados. Por sua vez, a pesquisa com finalidade clínica, envolvendo aspectos psíquicos e sua interferência no campo somático, não pode se dar fora do campo do singular.

Apesar das dificuldades em se construírem generalizações válidas (objetivo do conhecimento científico) baseadas no conhecimento oriundo do individual, este saber é fundamental para a efetiva manutenção e/ ou recuperação da saúde das pessoas reais (em oposição ao indivíduo abstrato da epidemiologia). Assim, a atenção às significações de uma gravidez para uma gestante em particular indicará aos profissionais de saúde que dela se ocupam as vias que, talvez somadas aos saberes já adquiridos e generalizáveis, tanto biológicos, como psicossociais, possam responder pela não-ocorrência de um parto prematuro. 


\section{Referências}

ALMEIDA FILHO, N., 1989. Epidemiologia sem Números. Uma Introdução Crítica à Ciência Epidemiológica. Rio de Janeiro: Editora Campus.

AMERICAN PSYCHIATRIC ASSOCIATION, 1994. Manual Diagnóstico e Estatístico dos Transtornos Mentais. São Paulo: Manole.

ANDREASEN, N. C., 1997. Linking Mind and Brain in the Study of Mental IIInesses: A Project for a Scientific Psychopathology. Science, 275:1586-1593.

ATLAN, H., 1991. Tudo, Não, Talvez. Educação eVerdade. Lisboa: Instituto Piaget.

BASTOS, F. I. P. \& CASTIEL, L. D., 1994. Epidemiologia e saúde mental no campo científico contemporâneo: labirintos que se entrecruzam? In: Psiquiatria Social e Reforma Psiquiátrica (P. Amarante, org.), pp. 97-111, Rio de Janeiro: Editora Fiocruz.

BUSTAN, M. N. \& COKER, A. L., 1994. Maternal attitude toward pregnancy and the risk of neonatal death. American Journal of Public Health, 84:411414.

CASTIEL, L. D., 1994. O Buraco e o Avestruz. A Singularidade do Adoecer Humano. Campinas: Papirus.

CLAVREUL, J., 1983. A Ordem Médica. Poder e Impotência do Discurso Médico. São Paulo: Editora Brasiliense.

COPPER, R. L.; GOLDENBERG, R. L.; DAS, A.; ELDER, N.; SWAIN, M.; NOEMAN, G.; RAMSEY, R.; COTRONEO, P.; COLLINS, B. A.; JOHNSON, F.; JONES, P. \& MEIER, A., 1996. The preterm prediction study: maternal estresse is associated with spontaneous preterm birth at less than thirty-five weeks' gestation. American Journal of Obstetrics and Gynecology, 175:1.286-1.292.

GRANGER, G. B., 1994. A Ciência e as Ciências. São Paulo: Editora Unesp.

HEDEGAARD, M.; HENRIKSEN, T. B.; SABROE, S. \& SECHER, N. J., 1993. Psychological distress in pregnancy and preterm delivery. British Medical Journal, 307:234-239.

HOBFOLL, S. E.; RITTER, C.; LAVIN, J.; HULSIZER, M R. \& CAMERON, R. P., 1995. Depression prevalence and incidence among inner-city pregnant and post partum women. Journal of Consulting and Clinical Psychology, 63:445-453.

JADRESIC, E.; JARA, C. \& ARAYA, R., 1993. Depression in pregnacy and puerperium: study of risk factors. Acta Psiquiatrica y Psicologica de América Latina, 39:63-74.

LAJEUNESSE, B. S., 1989. Em torno do DSM III. In: A Querela dos Diagnósticos (R. Gori, J. A. Miller \& R. Warkel, org.), pp. 70-73, Rio de Janeiro: Jorge Zahar Editor.

LAKOFF, G. \&JOHNSON, M., 1980. Metáforas dela Vida Cotidiana. Madrid: Cátedra.

LUTZ, C., 1985. Depression and translation of emotional worlds. In: Culture and Depression - Studies in the Anthropology and Cross-Cultural Psychiatry of Affect and Disorder (A. Kleinman \& B. Good, org.), pp. 63-100, Berkeley: University of California Press.
MILLAN, T.; YEVENEZ, R.; GALVEZ, M.; BAHAMONDE, M. I., 1990. A survey of the depressive symptoms in pregnant women at a urban primary care consultation office. Revista Médica de Chile, 118:1.230-1.234.

MORIN, E., 1990. Ciência com Consciência. Lisboa: Publicações Europa-América.

OMS (Organização Mundial da Saúde), 1993. Classificação dos Transtornos Mentais e de Comportamento da CID-10. Descrições Clínicas e Diretrizes Diagnósticas. Porto Alegre: Artes Médicas.

ORR, S. T. \& MILLER, C. A., 1995. Maternal depressive symptoms and the risk of poor pregnancy outcome. Review of the literature and preliminary findings. Epidemiogic Reviews, 17:65-171.

PALMEIRA, G. A. E. S., 1994. A Medida da Psique: Uma Apreciação das Variáveis Utilizadas na Investigação dos Aspectos Psi cológi cos Relacionados ao Câncer. Dissertação de Mestrado, Rio de Janeiro: Escola Nacional de Saúde Pública, Fundação Oswaldo Cruz.

PAYKEL, E. S., 1991. Depression in women. British Journal of Psychiatry, 158(Sup.10):22-29.

PEACOCK, J. L.; BLOUD, J. M . \& ANDERSON, H. R., 1995. Preterm delivery: effects of socioeconomic factors, psychological estresse, smoking, alchohol and caffeine. British Medical Journal, 311:531535.

SAM AJA, J., 1992. La combinación de métodos: pasos para una comprensión dialética del trabajo interdisciplinario. Educación Médica y Salud, 26:1-34.

SÉGUIN, L.; POTVIN, L. \& LOISELLE, J., 1995. Chronic estresseors, social support, and depression during pregnancy. Obstetrics and Gynecology, 4:583589.

SIM ÕES, R. D.; BARACAT, E. C.; HAIDAR, M. A.; PATRIARCA, M. T. \& LIMA, G. R., 1996. Estrogênio e depressão. Compacta Ginecológica, 11:4-6.

VICTORA, C. G.; BARROS, F. C.; HALPERN, R.; MENEZES, A. M. B.; HORTA, B. L.; TOMASI, E.; WEIDERPASS, E.; CASAR, J. A.; OLINTO, M. T.; GUIMARÃES, P. R. V.; GARCIA, M. M. \&VAUGHAN, J. P., 1996. Estudo longitudinal da população materno-infantil da região urbana do sul do Brasil, 1993: aspectos metodológicos e resultados preliminares. Revista de SaúdePública, 30:34-45.

ZARIFIAN, E., 1989. Um diagnóstico em psiquiatria: para quê? In: A Querela dos Diagnósticos (R. Gori, J. A. Miller \& R. Warkel, org.), pp. 45-51, Rio de Janeiro: Jorge Zahar Editor.

ZUCKERMAN, B.; AMARO, H.; BAUCHNER, H.; CABRAL, H., 1989. Depressive symptoms during pregnancy: relationship to poor health behavior. American Journal of Obstetrics and Gynecology, 160:1.107-1.111.

ZUCKERM AN, B.; BAUCHNER, H.; PARKER, S.; CABRAL, H., 1990. Maternal depressive symptons during pregnancy and newborn irritability. Journal of Develelopmental and Behavior Pediatrics, 11:190-194. 